\title{
Joint Signaling Strategies for Approaching the Capacity of Twisted-Pair Channels
}

\author{
Andrew Sendonaris, Student Member, IEEE, Venugopal V. Veeravalli, Member, IEEE, \\ and Behnaam Aazhang, Senior Member, IEEE
}

\begin{abstract}
A technique is presented for jointly optimizing the signaling in the two directions of transmission on a twistedpair communications channel. It is then applied to twisted-pair channel models with monotonic channel response and crosstalk transfer functions. While the signaling strategy presented in this paper can achieve only a lower bound on the true channel capacity, it is a significant improvement over existing signaling schemes. In particular, in contrast with existing schemes, the maximum information rate for the joint signaling strategy increases without bound as the signal-to-noise ratio (SNR) approaches infinity. It is also shown through numerical results that the proposed signaling strategy generalizes naturally to more practical nonmonotonic twisted-pair channel models incorporating bridge taps and other nonidealities. Finally, the form of the optimal signaling strategy suggests a relatively straightforward implementation using multicarrier modulation.
\end{abstract}

Index Terms - Capacity, crosstalk, digital subscriber loops, multicarrier modulation, twisted-pair channel.

\section{INTRODUCTION}

$\mathbf{R}$ ECENT WORK has shown that twisted-pair digital subscriber loops (DSL's) are capable of supporting very high data rates-in excess of $1.6 \mathrm{Mb} / \mathrm{s}$ within the standard carrier serving area (CSA), which typically covers a radius of about $12000 \mathrm{ft}$ around the central office [1]-[4]. In this paper we show that further significant increases in the capacity of twisted-pair loops can be achieved by jointly optimizing the signaling strategies for the two directions of transmission on the loops.

The following is a description of a typical DSL. Each twisted pair in a DSL is capable of supporting transmissions in both directions simultaneously, using an echo canceler [5]-[10]. Suppose there are $N$ twisted pairs in one binder cable ( $N$ usually equals 50 ). Then, each twisted pair has $N-1$ neighboring pairs which are transmitting information in both directions. At each end of a twisted pair, the received signal is corrupted by various noise sources, such as thermal noise from the electronics at the receiver, impulse noise from electromechanical switching devices at the central office, residual echo from the echo canceler, quantization noise, etc.

Paper approved by R. A. Kennedy, the Editor for Data Communications Modulation and Signal Design of the IEEE Communications Society. Manuscript received October 24, 1995; revised June 20, 1997 and December 15, 1997. This paper was presented in part at the 33rd Annual Allerton Conference on Communication, Control, and Computing, Monticello, IL, October 1995.

A. Sendonaris and B. Aazhang are with the Department of Electrical and Computer Engineering, Rice University, Houston, TX 77005 USA (e-mail: sendos@rice.edu; aaz@rice.edu).

V. V. Veeravalli is with the School of Electrical Engineering, Cornell University, Ithaca, NY 14853 USA (e-mail: venu@ee.cornell.edu).

Publisher Item Identifier S 0090-6778(98)03872-0.
For simplicity, these noise sources are modeled jointly as an additive white Gaussian noise (AWGN) source, whose power is chosen conservatively to compensate for possible inaccuracies in the model [3]. In addition, there is near-end crosstalk (NEXT) from the $N-1$ neighboring twisted pairs, which have transmitters that are physically close to the desired receiver and are sending information in the direction opposite to that of the received signal. There is also far-end crosstalk (FEXT), which is due to the transmission of information in the same direction as the received signal, that is, information sent by transmitters that are physically far from the desired receiver (see Fig. 1).

In asymmetrical digital subscriber line (ADSL) systems, each twisted pair has a low data-rate signal that is transmitted in a direction opposite to that of the main high data-rate signal. In such systems FEXT is significant and thus needs to be considered. However, the effects of FEXT are negligible compared to NEXT [4], [3] in most systems that support fullduplex transmission, such as high bit-rate digital subscriber lines (HDSL's), assuming that the loops are not too short. In this paper we will focus on the latter full-duplex DSL's.

In studying the information capacity of DSL's, all of the twisted pairs in a binder group can be considered jointly as a multiuser interference channel [11]. Obtaining the capacity region $^{1}$ of such a multiuser interference channel is, however, still an open problem even for the simplest case of two users, discrete time and additive Gaussian background noise [11]. To make the problem tractable, we focus on the portion of the capacity region corresponding to equal information rates for each of the users. The equal rate assumption is valid in the HDSL context since all users will have the same rate requirements.

Since signals that are transmitted in the same direction have negligible crosstalk between them, in the maximization of the bit rate, it is reasonable to assume that the power spectral densities (PSD's) of all of the signals transmitted in the same direction are equal to the same optimal PSD. An additional assumption that is made in much of the previous work on capacity calculations for twisted pairs is that the PSD's used in both directions of transmission are identical [12], [3], [13], [2]. The optimization to obtain the maximum bit rate for each twisted pair can then be performed over a single PSD. The capacity of each twisted-pair channel under the equal PSD (for both directions of transmission) constraint, and the

\footnotetext{
${ }^{1}$ The capacity region of a multiuser channel is the set of all possible combinations of achievable user rates.
} 


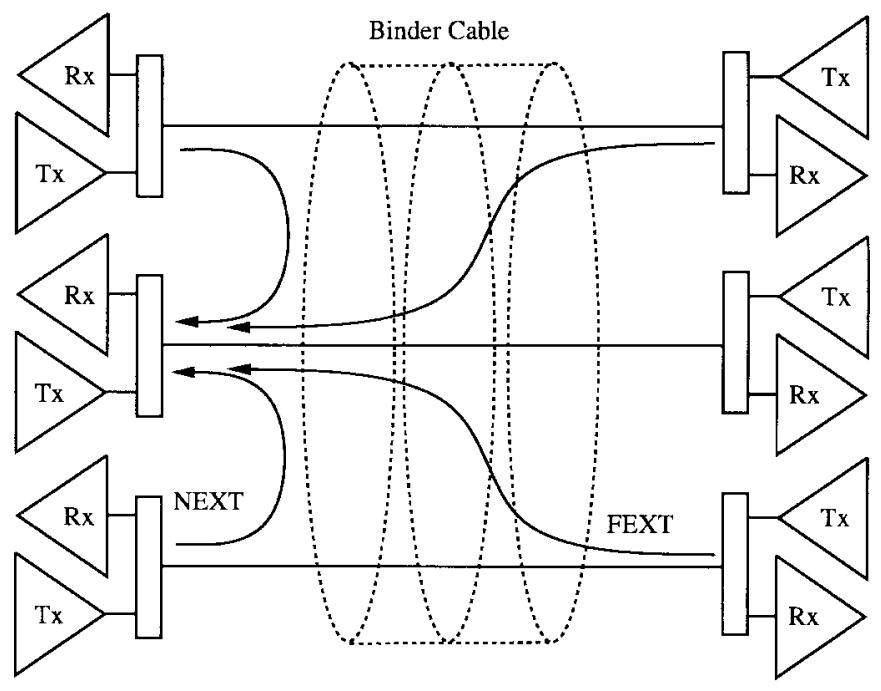

Fig. 1. Schematic representation of NEXT and FEXT in a DSL.

corresponding maximizing signal PSD have been derived [12], [3]. However, as noted in these papers, the capacity of each twisted pair under the equal PSD constraint converges to a finite constant value as the signal-to-(background)noise ratio (SNR) goes to infinity. This fact is an indication that the equal PSD constraint is too restrictive. If, for example, the signals in the two directions of transmissions are chosen to have PSD's that occupy disjoint frequency bands in the available bandwidth, the capacity of each twisted pair will be unbounded as the SNR approaches infinity.

In recent work, Lechleider [14] notes that the equal PSD constraint leads to decreased capacity and further calculates the capacity without this constraint. However, in the analysis in [14] it is assumed that the PSD's have the same support-an assumption that rules out the possibility that the signals have disjoint spectra. The results given in [14] show only a slight improvement in capacity over the equal PSD case.

In this paper we show that significant gains in capacity can be obtained by removing the equal PSD constraint. The capacity obtained with the optimal signaling strategy can be far larger than the equal PSD capacity; furthermore, it goes to infinity as the SNR approaches infinity.

This paper is organized as follows. In Section II the capacity problem is formulated as the maximization of the bit rate over the PSD's for the two directions of transmission. The optimal signal PSD's and corresponding capacity are obtained for a simplified channel model in Section III. This result is then extended to a general channel model in Section IV, and numerical results that corroborate the analysis are given in Section V. Conclusions are given in Section VI.

\section{PRoblem Formulation}

As mentioned in Section I, we assume that each direction of transmission will have its own PSD, say $S_{1}(f)$ and $S_{2}(f)$. Let direction 1 refer to the direction of transmission using $S_{1}(f)$ as its PSD, and let direction 2 refer to the other direction. The channel is assumed to have transfer function $H_{c}(f)$, and two sources of noise: AWGN with one-sided spectral density
$\mathcal{N}_{o}$, and NEXT with crosstalk transfer function $H_{x}(f)$. For the purposes of capacity calculations, it is shown in [15] that crosstalk can be modeled as Gaussian noise without substantial error. We use the Gaussian crosstalk assumption in our analysis. Under these assumptions, it is easily shown that the capacity for direction 1 , in bits per second, is given by (see [12], [3], [14])

$$
C_{1}=\sup _{S_{1}(f), S_{2}(f)} \int_{0}^{\infty} \log _{2}\left[1+\frac{\left|H_{c}(f)\right|^{2} S_{1}(f)}{\mathcal{N}_{o}+\left|H_{x}(f)\right|^{2} S_{2}(f)}\right] d f
$$

where the supremum is taken over all $S_{1}(f)$ and $S_{2}(f)$ such that

$$
\begin{aligned}
S_{i}(f) & \geq 0 \quad \forall f \\
\int_{0}^{\infty} S_{i}(f) d f & \leq P_{\max }, \quad \text { for } i=1,2
\end{aligned}
$$

and where $S_{i}(f)$ is the PSD of direction $i$, and the latter inequality corresponds to an average power constraint.

The capacity for direction 2 is given by the analogous expression. Of course, when designing the transmission scheme for both directions, it becomes necessary to consider them simultaneously. As can be seen from (1), when only direction 1 is considered, $C_{1}$ is achieved when $S_{2}(f)=0 \forall f$, which implies that $C_{2}=0$. The same problem arises when only direction 2 is considered. Therefore, a joint optimization needs to be performed. There are three ways in which this can be done

$$
\begin{array}{ll}
\operatorname{maximize} & C_{1} \text { subject to the constraint } C_{1}=C_{2} \\
\text { maximize } & C_{1}+C_{2} \\
\text { maximize } & \min \left\{C_{1}, C_{2}\right\} .
\end{array}
$$

Let

$$
\tilde{C}\left(S_{1}(f), S_{2}(f)\right)=\int_{0}^{\infty} \log _{2}\left[1+\frac{\left|H_{c}(f)\right|^{2} S_{1}(f)}{\mathcal{N}_{o}+\left|H_{x}(f)\right|^{2} S_{2}(f)}\right] d f .
$$

Then the capacities resulting from the joint optimization are given by

$$
C_{1}=\tilde{C}\left(S_{1}^{*}(f), S_{2}^{*}(f)\right) \quad C_{2}=\tilde{C}\left(S_{2}^{*}(f), S_{1}^{*}(f)\right)
$$


where $S_{1}^{*}(f)$ and $S_{2}^{*}(f)$ are chosen according to one of the three criteria given in (2)-(4).

All three approaches to joint optimization are analytically intractable without constraints on the PSD's. An approach to solving for $C_{1}$ and $C_{2}$ that appears in much of the literature is to assume that the PSD's are the same in both directions of transmission, i.e., $S_{1}(f)=S_{2}(f)$. Under this constraint, the joint optimization problem to obtain capacity [under all three criteria given in (2)-(4)] reduces to [12], [3]

$$
C_{1}=\max _{S_{1}(f)} \tilde{C}\left(S_{1}(f), S_{1}(f)\right) \text {. }
$$

However, as discussed in Section I, the equal PSD assumption is too restrictive, and significant gains in capacity are possible by removing this assumption. The main idea of this paper is to make $S_{1}(f)$ and $S_{2}(f)$ "symmetric," in some sense, thus reducing the optimization problem for direction 1 to

$$
C_{1}=\max _{S_{1}(f)} \tilde{C}\left(S_{1}(f), S_{1}^{\mathrm{sym}}(f)\right)
$$

where $S_{1}^{\mathrm{sym}}(f)$ is symmetric to $S_{1}(f)$. Despite the fact that it is difficult to rigorously define the concept of symmetry, generally speaking, it implies that $S_{1}^{\mathrm{sym}}(f)$ is small where $S_{1}(f)$ is large, and vice versa, so that $\tilde{C}\left(S_{1}(f), S_{1}^{\mathrm{sym}}(f)\right)=$ $\tilde{C}\left(S_{1}^{\text {sym }}(f), S_{1}(f)\right)$. Hence, under the symmetry assumption, the three criteria for joint optimization given in (2)-(4) are equivalent.

The second idea is to parameterize $S_{1}(f)$ so that the maximization is taken over the parameters that now describe $S_{1}(f)$. Both of these ideas are first described for a simplified channel model.

\section{SIMPLIFIED CHANNEL}

This section considers a simple channel with a constant channel transfer function and a constant crosstalk transfer function. The objective is, after finding the optimal $S_{1}(f)$ and $S_{2}(f)$ for this channel, to extend the result to the generalized twisted-pair channel.

Assume a channel with

$$
\begin{aligned}
\left|H_{c}(f)\right|^{2} & = \begin{cases}H, & \text { if }|f| \leq W \\
0, & \text { otherwise }\end{cases} \\
\left|H_{x}(f)\right|^{2} & = \begin{cases}X, & \text { if }|f| \leq W \\
0, & \text { otherwise. }\end{cases}
\end{aligned}
$$

Consider the class of $S_{1}(f)$ and $S_{2}(f)$ defined as follows:

$$
\begin{aligned}
& S_{1}(f)=\left\{\begin{array}{l}
\alpha \frac{2 P_{\max }}{W}, \quad \text { if }|f| \leq \frac{W}{2} \\
(1-\alpha) \frac{2 P_{\max }}{W}, \quad \text { if } \frac{W}{2}<|f| \leq W \\
0, \quad \text { otherwise }
\end{array}\right. \\
& S_{2}(f)= \begin{cases}(1-\alpha) \frac{2 P_{\max }}{W}, & \text { if }|f| \leq \frac{W}{2} \\
\alpha \frac{2 P_{\max }}{W}, & \text { if } \frac{W}{2}<|f| \leq W \\
0, & \text { otherwise. }\end{cases}
\end{aligned}
$$

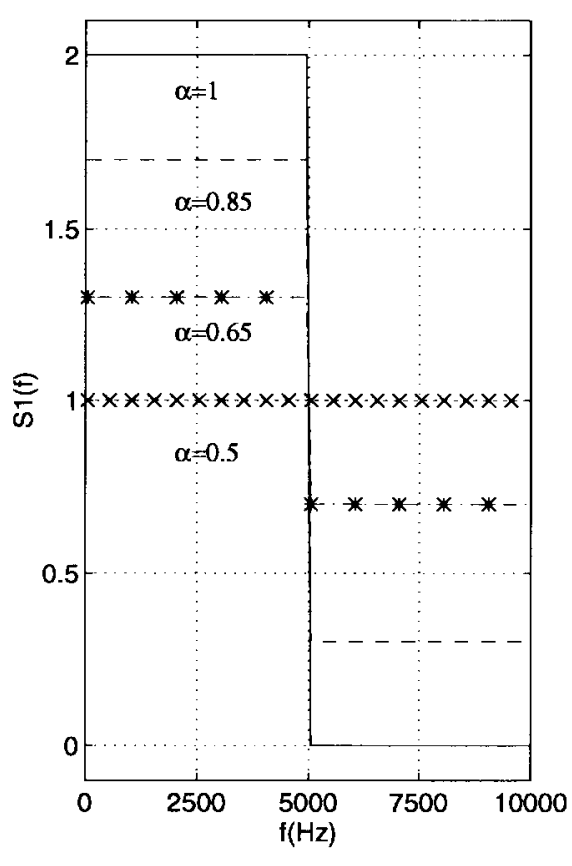

(a)

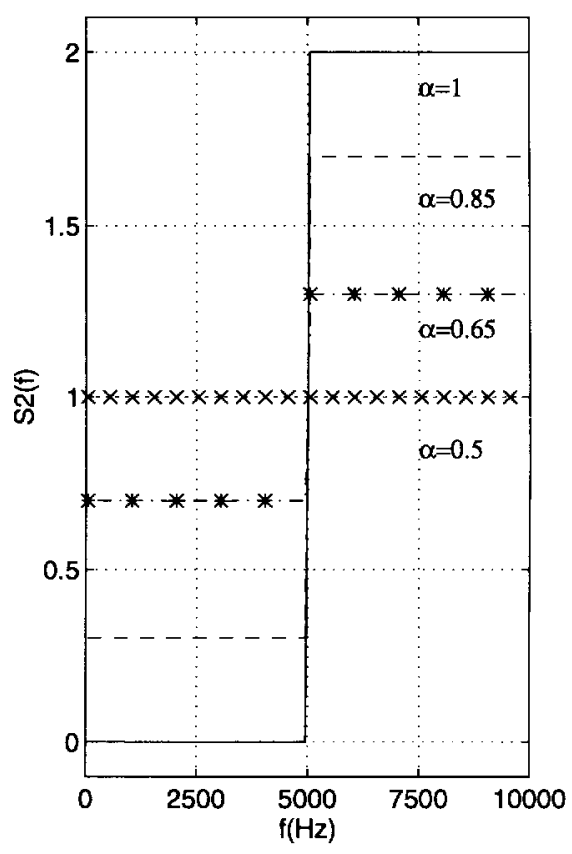

(b)

Fig. 2. Simplified channel: variations of (a) $S_{1}(f)$ and (b) $S_{2}(f)$ with $\alpha$.

where $0.5 \leq \alpha \leq 1$ (see Fig. 2). In this configuration $\alpha=0.5$ corresponds to using equal PSD's for the two directions and $\alpha=1$ corresponds to using frequencydivision signaling (FDS). By construction of $S_{1}(f)$ and $S_{2}(f), C_{1}={ }^{\sim} C\left(S_{1}(f), S_{2}(f)\right)={ }^{\sim} C\left(S_{2}(f)\right.$, $\left.S_{1}(f)\right)=C_{2}$. Therefore, $S_{1}(f)$ and $S_{2}(f)$, as defined above, are symmetric in the sense described in Section II. It is easily verified that $S_{1}(f)$ and $S_{2}(f)$ satisfy the power constraint. The resulting capacity for 
direction 1 becomes

$$
\begin{aligned}
C_{1}= & \max _{0.5 \leq \alpha \leq 1} \frac{W}{2} \log _{2}\left[1+\frac{\alpha \frac{2 P_{\max }}{W} H}{\mathcal{N}_{o}+(1-\alpha) \frac{2 P_{\max }}{W} X}\right] \\
& +\frac{W}{2} \log _{2}\left[1+\frac{(1-\alpha) \frac{2 P_{\max }}{W} H}{\mathcal{N}_{o}+\alpha \frac{2 P_{\max }}{W} X}\right] \\
= & \max _{0.5 \leq \alpha \leq 1} \frac{W}{2 \ln (2)}\left\{\ln \left[1+\frac{\alpha G H}{1+(1-\alpha) G X}\right]\right. \\
& \left.+\ln \left[1+\frac{(1-\alpha) G H}{1+\alpha G X}\right]\right\}
\end{aligned}
$$

where $G=2 P_{\max } / \mathcal{N}_{o} W$.

Due to the symmetry of $S_{1}(f)$ and $S_{2}(f), C_{2}$ will be equal to $C_{1}$ for any value of $\alpha$. Therefore, it is only necessary to maximize $C_{1}$. Taking the derivative with respect to $\alpha$

$$
\begin{aligned}
\frac{\partial \tilde{C}}{\partial \alpha}= & \frac{W}{2 \ln (2)}\left[\frac{G H(1+G X)}{(1+(1-\alpha) G X+\alpha G H)(1+(1-\alpha) G X)}\right. \\
& \left.-\frac{G H(1+G X)}{(1+\alpha G X+(1-\alpha) G H)(1+\alpha G X)}\right] \\
= & \frac{W}{2 \ln (2)} \frac{(2 \alpha-1)\left(2 X-H+G X^{2}\right) G^{2} H(1+G X)}{(1+(1-\alpha) G X+\alpha G H)(1+(1-\alpha) G X)} \\
& \cdot \frac{1}{(1+\alpha G X+(1-\alpha) G H)(1+\alpha G X)} \\
= & (2 \alpha-1)\left(2 X-H+G X^{2}\right) Q
\end{aligned}
$$

where $Q$ is always a positive quantity.

Therefore, $\alpha=1 / 2$ is the only stationary point. If it is a maximum, it is optimum to use equal PSD's for the two directions of transmission. If it is a minimum, then $\alpha=1$ achieves the maximum, which means that it is optimum to use FDS for the two directions.

For all $\alpha>0.5$, the derivative of $\tilde{C}$ with respect to $\alpha$ will be negative if and only if $2 X-H+G X^{2}<0$. This implies that $\alpha=1 / 2$ is a maximum if and only if $G<(H-2 X) / X^{2}$. Similarly, $\alpha=1 / 2$ is a minimum if and only if $G>(H-2 X) / X^{2}$. Thus, since $G=2 P_{\max } / \mathcal{N}_{o} W$, it follows that

$$
\frac{2 P_{\max }}{\mathcal{N}_{o} W} \underset{\text { fds }}{\stackrel{\text { eqpsd }}{<}} \frac{H-2 X}{X^{2}}
$$

where eqpsd denotes using equal PSD's for the two directions of transmission and fds denotes using frequency division.

It should be noted that the capacity of the channel obtained by using $S_{1}(f)$ and $S_{2}(f)$ according to (8) is only a lower bound on the true capacity of the channel since we are constraining the form of $S_{1}(f)$ and $S_{2}(f)$ according to (7). However, there are indications that the threshold test given in (8) achieves the global maximum more generally. For example, if the constraint that $S_{1}(f)$ and $S_{2}(f)$ have to be symmetric is relaxed, a new class of $S_{1}(f)$ and $S_{2}(f)$ can be

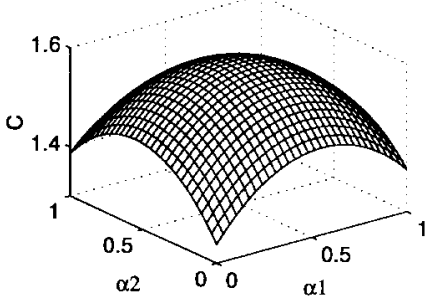

(a)

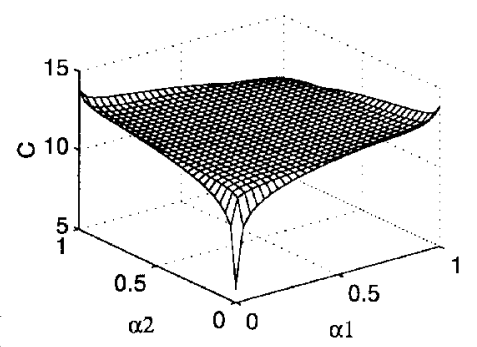

(c)

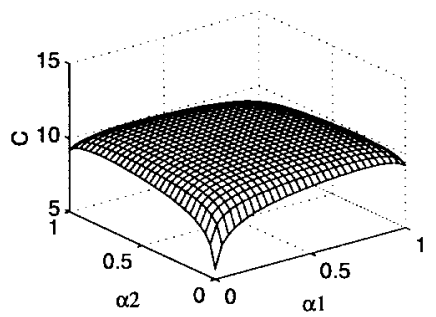

(b)

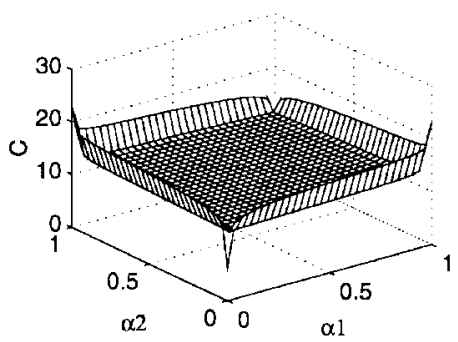

(d)
Fig. 3. $C_{1}+C_{2}$ as a function of $\alpha_{1}$ and $\alpha_{2}$, for four different values of SNR. (a) $\mathrm{SNR}=1$. (b) SNR $=100$. (c) $\mathrm{SNR}=1000$. (d) $\mathrm{SNR}=1 \mathrm{e}+05$.

defined as follows:

$$
S_{1}(f)=\left\{\begin{array}{l}
\alpha_{1} \frac{2 P_{\max }}{W}, \quad \text { if }|f| \leq \frac{W}{2} \\
\left(1-\alpha_{1}\right) \frac{2 P_{\max }}{W}, \quad \text { if } \frac{W}{2}<|f| \leq W \\
0, \quad \text { otherwise }
\end{array}\right.
$$

$$
S_{2}(f)= \begin{cases}\alpha_{2} \frac{2 P_{\max }}{W}, & \text { if }|f| \leq \frac{W}{2} \\ \left(1-\alpha_{2}\right) \frac{2 P_{\max }}{W}, & \text { if } \frac{W}{2}<|f| \leq W \\ 0, \quad \text { otherwise. } & \end{cases}
$$

That is, both $S_{1}(f)$ and $S_{2}(f)$ have the form shown in Fig. 2 , however, they are not forced to be coupled; that is, $\alpha_{2}$ is not set to be equal to $1-\alpha_{1}$, as in (7). In this case, when maximizing the sum of the capacities of directions 1 and 2, it can be shown that $\alpha_{1}=\alpha_{2}=1 / 2$ is the only stationary point and thus can be a maximum, a minimum, or a saddle point. If it is a maximum, then using equal PSD's for the two directions is optimal. If it is not a maximum, the maximum will be achieved at one of the corner solutions, i.e., at $\alpha_{1}=1$, $\alpha_{2}=0$ or $\alpha_{1}=0, \alpha_{2}=1$, which corresponds to using FDS ( $\alpha_{1}=\alpha_{2}=0$ and $\alpha_{1}=\alpha_{2}=1$ can easily be shown to not achieve the maximum). Therefore, only equal PSD's and FDS can be optimal, which leads to the same threshold test as in (8). Fig. 3(a) and (b) plots the sum of the capacities of directions 1 and 2, versus all possible combinations of $\alpha_{1}$ and $\alpha_{2}$, for a given channel and for four different values of SNR. Notice how the point $\alpha_{1}=\alpha_{2}=1 / 2$ changes from a maximum to a saddle point as the SNR increases.

As a second example, we consider changing the form of $S_{1}(f)$ and $S_{2}(f)$, while keeping the constraint that $S_{1}(f)$ and $S_{2}(f)$ are symmetric. That is, we consider the class of $S_{1}(f)$ 


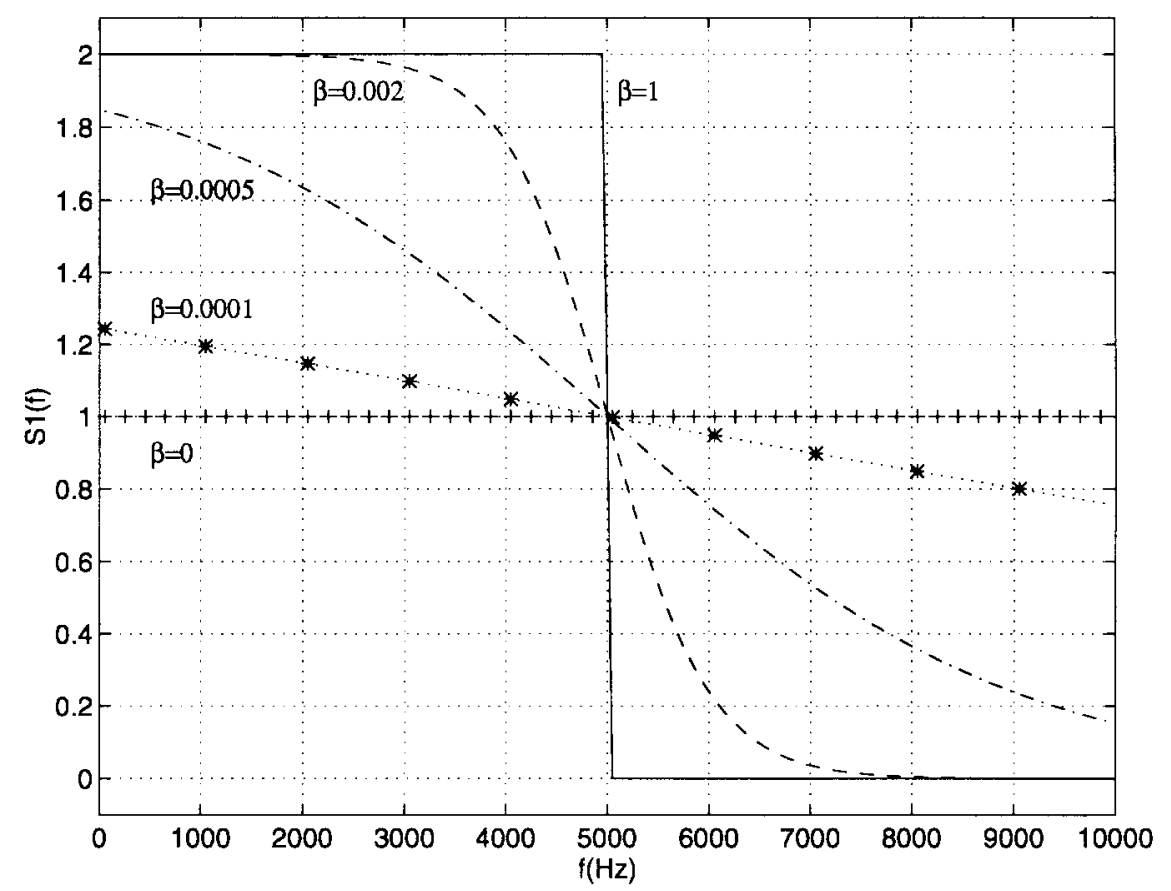

Fig. 4. Simplified channel: variation of $S_{1}(f)$ with $\beta$.

and $S_{2}(f)$ defined as follows:

$$
\begin{aligned}
& S_{1}(f)= \begin{cases}\frac{2 P_{\max }}{W} \frac{1}{1+\exp \left(\beta\left(|f|-\frac{W}{2}\right)\right)}, & \text { if }|f| \leq W \\
0, & \text { otherwise }\end{cases} \\
& S_{2}(f)= \begin{cases}\frac{2 P_{\max }}{W} \frac{1}{1+\exp \left(-\beta\left(|f|-\frac{W}{2}\right)\right)}, & \text { if }|f| \leq W \\
0, & \text { otherwise }\end{cases}
\end{aligned}
$$

where $0 \leq \beta \leq \infty$ (see Fig. 4). In this configuration $\beta=0$ corresponds to using equal PSD's for the two directions of transmission and $\beta=\infty$ corresponds to using FDS. It can easily be shown that, as defined above, $S_{1}(f)$ and $S_{2}(f)$ are symmetric. That is, $C_{1}=C_{2}$ for all values of $\beta$. Fig. 5 plots the capacity of directions 1 and 2 , as a function of $\beta$, for a given channel and for four different values of SNR. Notice how the point $\beta=0$ changes from a maximum to a minimum as the SNR increases from a value less than $(H-2 X) / X^{2}$ to a value greater than $(H-2 X) / X^{2}$, indicating that the threshold test in (8) still applies.

\section{Generalized Channel}

Consider a channel that is bandlimited to $|f| \leq W$ but is no longer restricted to being constant over this frequency range. The frequency range $0 \leq f \leq W$ is divided ${ }^{2}$ into $M$ equal-width bins ( $M$ parallel independent subchannels). Within each bin, both $H_{c}(f)$ and $H_{x}(f)$ will be approximately constant, given that $M$ is sufficiently large. Also, any capacitymaximizing scheme will have to decide how much power to allocate for each bin. Therefore, using the results from the

\footnotetext{
${ }^{2}$ Since the capacity formula in (1) involves only positive frequencies, only the frequency range $0 \leq f \leq W$ will be considered henceforth.
}

previous section, it follows that in the $i$ th bin (with center frequency $f_{i}$ ), the optimum power allocation rule is given by

$$
\frac{2 P_{i}}{\mathcal{N}_{0} D} \underset{\text { fds }}{\stackrel{\text { eqpsd }}{<}} \frac{H_{i}-2 X_{i}}{X_{i}^{2}}
$$

where $D=W / M, H_{i}=H_{c}\left(f_{i}\right), X_{i}=H_{x}\left(f_{i}\right), f_{i}=$ $D / 2+(i-1) D$. Therefore, the capacity of this channel is given by the solution to the following optimization problem:

$$
C\left(P_{\max }\right)=\max _{\substack{P_{i}, i=1,2, \cdots, M \\ \text { s.t. } 0 \leq P_{i} \leq P_{\max }}} \sum_{i=1}^{M} C_{\mathrm{bin}}\left(P_{i}, H_{i}, X_{i}, \mathcal{N}_{o}, D\right)
$$

where

$$
\begin{aligned}
& C_{\mathrm{bin}}\left(P_{i}, H_{i}, X_{i}, \mathcal{N}_{o}, D\right) \\
& =\left\{\begin{array}{cl}
D \log _{2}\left(1+\frac{P_{i} H_{i}}{N_{o} D+P_{i} X_{i}}\right), & \text { if } \frac{2 P_{i}}{\mathcal{N}_{o} D} \leq \frac{H_{i}-2 X_{i}}{X_{i}^{2}} \\
\frac{D}{2} \log _{2}\left(1+\frac{2 P_{i} H_{i}}{N_{o} D}\right), & \text { if } \frac{2 P_{i}}{\mathcal{N}_{o} D}>\frac{H_{i}-2 X_{i}}{X_{i}^{2}}
\end{array}\right.
\end{aligned}
$$

Alternatively, the problem can also be posed as follows:

$$
C\left(P_{\max }\right)=\max _{\substack{\mathcal{A}, P_{\mathcal{A}} \\ \text { s.t. } \mathcal{A} \subset\{1,2,3, \cdots, M\} \\ 0 \leq P_{\mathcal{A}} \leq P_{\max }}} C_{\text {eqpsd }}\left(\mathcal{A}, P_{\mathcal{A}}\right)+C_{\mathrm{fds}}\left(\mathcal{A}^{\mathcal{C}}, P_{\max }-P_{\mathcal{A}}\right)
$$

where $\mathcal{A}$ is the set of indexes of the bins that will employ equal PSD and $P_{\mathcal{A}}$ is the power allocated to this collection of bins. Also, $C_{\text {eqpsd }}\left(\mathcal{A}, P_{\mathcal{A}}\right)$ denotes the capacity of a channel whose transfer function consists of only the bins included in 


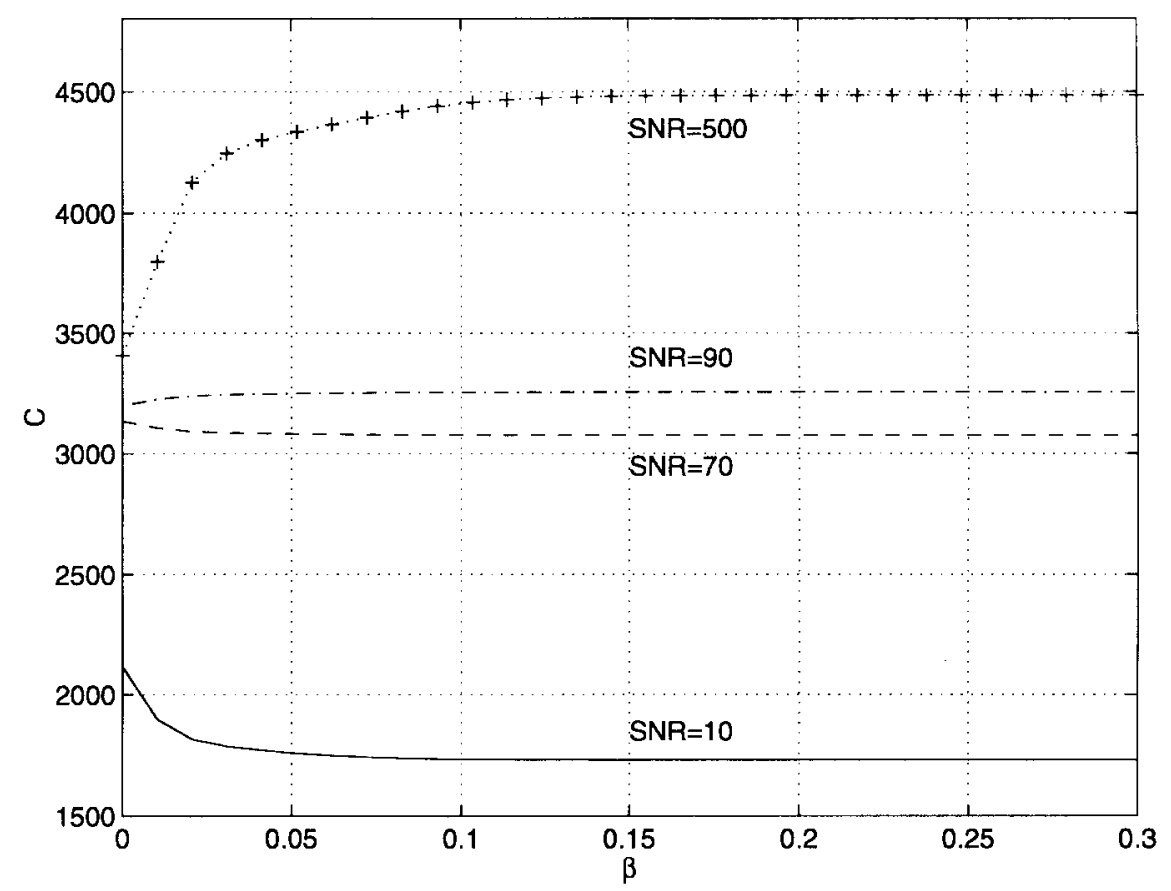

Fig. 5. $C_{1}$ (and $\left.C_{2}\right)$ as a function of $\beta$, for four different values of SNR. In this example, $\left((H-2 X) / X^{2}\right)=80$.

$\mathcal{A}$, when the power constraint is $P_{\mathcal{A}}$, and when equal PSD's are used for both directions of transmission. It is evident that the remaining bins, included in $\mathcal{A}^{\mathcal{C}}$, will employ frequency division and use a total power equal to $P_{\max }-P_{\mathcal{A}}$. The capacity of a channel whose transfer function consists of only the bins included in $\mathcal{B}$, has a power constraint equal to $P_{\mathcal{B}}$, and employs FDS for both directions of transmission, is denoted by $C_{\mathrm{fds}}\left(\mathcal{B}, P_{\mathcal{B}}\right)$. This channel effectively suffers only from AWGN. Both $C_{\text {eqpsd }}\left(\mathcal{A}, P_{\mathcal{A}}\right)$ and $C_{\text {fds }}\left(\mathcal{B}, P_{\mathcal{B}}\right)$ are well studied and well described in the literature [12], [3].

Unfortunately, as $M$ increases, the optimization problems described by (11) and (13) both become prohibitively computationally complex. In fact, the problem described in (13) falls under the category of mixed-integer programming, which is still an open research area.

There are cases, though, when the problem in (13) can be reduced to a significantly simpler one. This occurs, for example, when $H_{c}(f)$ is a decreasing function of $f$ and $H_{x}(f)$ is an increasing function of $f$. A decreasing $H_{c}(f)$ and an increasing $H_{x}(f)$ result in a decreasing $\left(H_{c}(f)-\right.$ $\left.2 H_{x}(f)\right) / H_{x}^{2}(f)$, which implies that $\left(H_{i}-2 X_{i}\right) / X_{i}^{2}$ is a decreasing function of $i$. Thus, if there exists an $i^{*}$ such that $\left(H_{i^{*}}-2 X_{i^{*}}\right) / X_{i^{*}}^{2}<0$, then $\left(H_{i}-2 X_{i}\right) / X_{i}^{2}<0 \forall i \geq$ $i^{*}$. Since $P_{i} \geq 0$ for all $i$, it follows that $2 P_{i} / \mathcal{N}_{0} D>$ $\left(H_{i}-2 X_{i}\right) / X_{i}^{2} \forall i \geq i^{*}$, which in turn implies that FDS is always optimum for all bins after (and including) the $i^{*}$ th bin, according to (10).

Therefore, the capacity-maximizing scheme must use FDS $\forall i \geq i^{*}$. Assume that as $i$ decreases from $i^{*}$ to 1 , the strategy changes from frequency division to equal PSD's. That is, $\exists M_{o}$, in the range $1 \leq M_{o}<i^{*}$, such that equal PSD's are used in the $M_{0}$ th bin and frequency division is used in all bins to the right of the $M_{O}$ th bin. Theorem 1 shows that, in such a case, the capacity-maximizing scheme will use equal PSD's for all bins from 1 to $M_{0}$.

Lemma 1: Let

$$
\begin{aligned}
& \left|H_{c}(f)\right|^{2}= \begin{cases}H_{1}, & \text { if } 0 \leq|f| \leq \frac{W}{2} \\
H_{2}, & \text { if } \frac{W}{2}<|f| \leq W \\
0, & \text { otherwise }\end{cases} \\
& \left|H_{x}(f)\right|^{2}= \begin{cases}X_{1}, & \text { if } 0 \leq|f| \leq \frac{W}{2} \\
X_{2}, & \text { if } \frac{W}{2}<|f| \leq W \\
0, & \text { otherwise. }\end{cases}
\end{aligned}
$$

Assume that there is a power constraint on the transmitted signal. Then, if $H_{i}-2 X_{i}>0$ for $i=1,2, H_{1}>H_{2}$, and $X_{1}<X_{2}$, a higher capacity is always achieved when using equal PSD's in bin 1 and FDS in bin 2 than when using FDS in bin 1 and equal PSD's in bin 2.

The proof of Lemma 1 is lengthy and involved and does not permit inclusion in this paper. However, the proof can be found in [16].

Theorem 1: Let $H_{c}(f)$ be a decreasing and $H_{x}(f)$ an increasing function of $f$. Consider the class of all $M$-bin transmission schemes, that is, schemes that consist of dividing the given frequency range into $M$ bins and, within each bin, deciding to perform equal PSD's or FDS. The capacitymaximizing scheme will use equal PSD's for bins 1 to $M_{o}$, and frequency division for bins $M_{o}+1$ to $M$, for some $0 \leq M_{\circ} \leq M$.

Proof: First, it is convenient to define $e_{i}$ to indicate that equal PSD's will be used in bin $i$, and to define $f_{i}$ to indicate that FDS will be used in bin $i$. 
As was argued previously, the capacity-maximizing scheme will have the following form:

$$
\cdots, e_{M_{o}}, f_{M_{o}+1}, f_{M_{o}+2}, \cdots, f_{M}
$$

for some $0 \leq M_{o} \leq M$. The transmission strategies for bins $M_{O}-1$ to 1 are yet to be determined. Assume that as $i$ decreases from $M_{O}$ to 1 , the strategy changes from equal PSD's to FDS. That is, assume that for some $i_{o}, 1 \leq i_{o}<M_{o}$, the following is true about the capacity-maximizing scheme:

$$
\cdots, f_{i_{o}}, e_{i_{o}+1}, e_{i_{o}+2}, \cdots, e_{M_{o}-1}, e_{M_{o}}, f_{M_{o}+1}, f_{M_{o}+2}, \cdots, f_{M}
$$

Then, capacity can always be increased by switching the transmission strategy in bins $i_{o}$ and $M_{O}$ while keeping the total power in both bins the same. That is, the following scheme:

$$
\cdots, e_{i_{o}}, e_{i_{o}+1}, e_{i_{o}+2}, \cdots, e_{M_{o}-1}, f_{M_{o}}, f_{M_{o}+1}, f_{M_{o}+2}, \cdots, f_{M}
$$

has a higher capacity due to Lemma 1 . However, this leads to a contradiction because the scheme described in (14) was assumed to maximize capacity. Thus, there is no $i_{o}, 1 \leq i_{o}<$ $M_{O}$ such that the transmission strategy changes from equal PSD's to FDS, which implies that the capacity-maximizing scheme will have the following form:

$$
e_{1}, e_{2}, \cdots, e_{M_{o}-2}, e_{M_{o}-1}, e_{M_{o}}, f_{M_{o}+1}, f_{M_{o}+2}, \cdots, f_{M}
$$

This result is quite intuitive-let both directions of transmission utilize the "good" parts of the channel (where crosstalk is low), and use frequency division to eliminate crosstalk in portions of the channel where the crosstalk is high.

Therefore $C\left(P_{\max }\right)$ is as given in (15), shown at the bottom of the page. As mentioned previously, techniques for computing both $C_{\text {eqpsd }}$ and $C_{\mathrm{fds}}$ have been given by other researchers [12], [3]. However, closed-form expressions for $C_{\text {eqpsd }}$ and $C_{\text {fds }}$ are not obtainable in general. Therefore, the maximization in (15) can, in general, only be performed numerically. Examples of this maximization are given in Section V. Note also that $C\left(P_{\max }\right)$ is only a lower bound on the true capacity of the channel since we are constraining the form of $S_{1}(f)$ and $S_{2}(f)$.

\section{A. Bridge Taps}

In practical twisted-pair channels, bridge taps and other nonidealities introduce nulls at certain parts of the spectrum and violate the assumption of monotonic channel and crosstalk transfer functions. A system designer could, of course, require the system to not have bridge taps, and could alleviate the other nonidealities, thus achieving monotonicity of the channel and crosstalk transfer functions. In such a case, Theorem 1 would be directly applicable.

However, even if we have no control over a system with nonmonotonic transfer functions, the analysis carried out so far can be used in a constructive manner. Firstly, it is clear that, if we use a monotonic channel model that uniformly bounds the actual channel transfer functions, we obtain an upper bound on the actual capacity. Secondly, and more significantly, even though a result corresponding to Theorem 1 for nonmonotonic channels is at present elusive, the form of the capacitymaximizing scheme in Theorem 1 allows us to conjecture a solution structure for the nonmonotonic channels. The key idea in Theorem 1 is that equal PSD's should be used in the bins where the channel is "good" and frequency division should be used where the crosstalk becomes a problem. Therefore, we would expect to see the following behavior for a general nonmonotonic channel as the SNR varies. At low SNR's, all bins with positive $\left(H_{i}-2 X_{i}\right) / X_{i}^{2}$ will employ equal PSD's, whereas bins where this quantity is negative will employ frequency division. As the SNR increases, we expect to see the equal PSD regions interspersed by pockets of frequencydivision bins. These pockets will form in the parts of the spectrum where the nulls in the channel transfer function are. As the SNR increases even further, these pockets of frequencydivision bins will become wider and wider until all but the "best" bins will employ frequency division. Numerical results in Section V show that this is indeed the case.

\section{Numerical Results AND Discussion}

The capacity $C\left(P_{\max }\right)$ was numerically calculated, using (15), for two monotonic twisted-pair channels: one studied in [12] and one studied in [3]. The model of the twisted-pair channel used in [12] is

$$
\left|H_{c}(f)\right|^{2}=\exp (-\alpha \sqrt{f})
$$

where $\alpha=k\left(l / l_{o}\right)$, the length of the channel is $l \mathrm{ft}, l_{o}$ is the reference length of $18000 \mathrm{ft}$, and $k$ is a constant of the physical channel and is equal to 1.158. Also,

$$
\left|H_{x}(f)\right|^{2}=\beta f^{3 / 2}
$$

where $\beta=10^{-9}$. In the above expressions, $f$ refers to frequency in kilohertz. The capacity of this channel was calculated using $l=600 \mathrm{ft}, W=100 \mathrm{MHz}$, and $M=$ 1000. The values used for $l$ and $W$ correspond to the ones depicted in [12], and the value used for $M$ was chosen such that no significant change in calculated capacity resulted from increasing it. The resulting capacity, along with the corresponding threshold frequency $f_{M_{o}}$, are shown in Fig. 6 as functions of the SNR, which is defined as SNR = $2 P_{\max } / \mathcal{N}_{o} W$.

$$
C\left(P_{\max }\right)=\max _{\substack{M_{o}, P_{1} \\ \text { s.t. } 0 \leq M_{o} \leq M \\ 0 \leq P_{1} \leq P_{\max }}} C_{\text {eqpsd }}\left(\left\{i \mid i \in\left(0, M_{o}\right]\right\}, P_{1}\right)+C_{\mathrm{fds}}\left(\left\{i \mid i \in\left(M_{o}, M\right]\right\}, P_{\max }-P_{1}\right)
$$




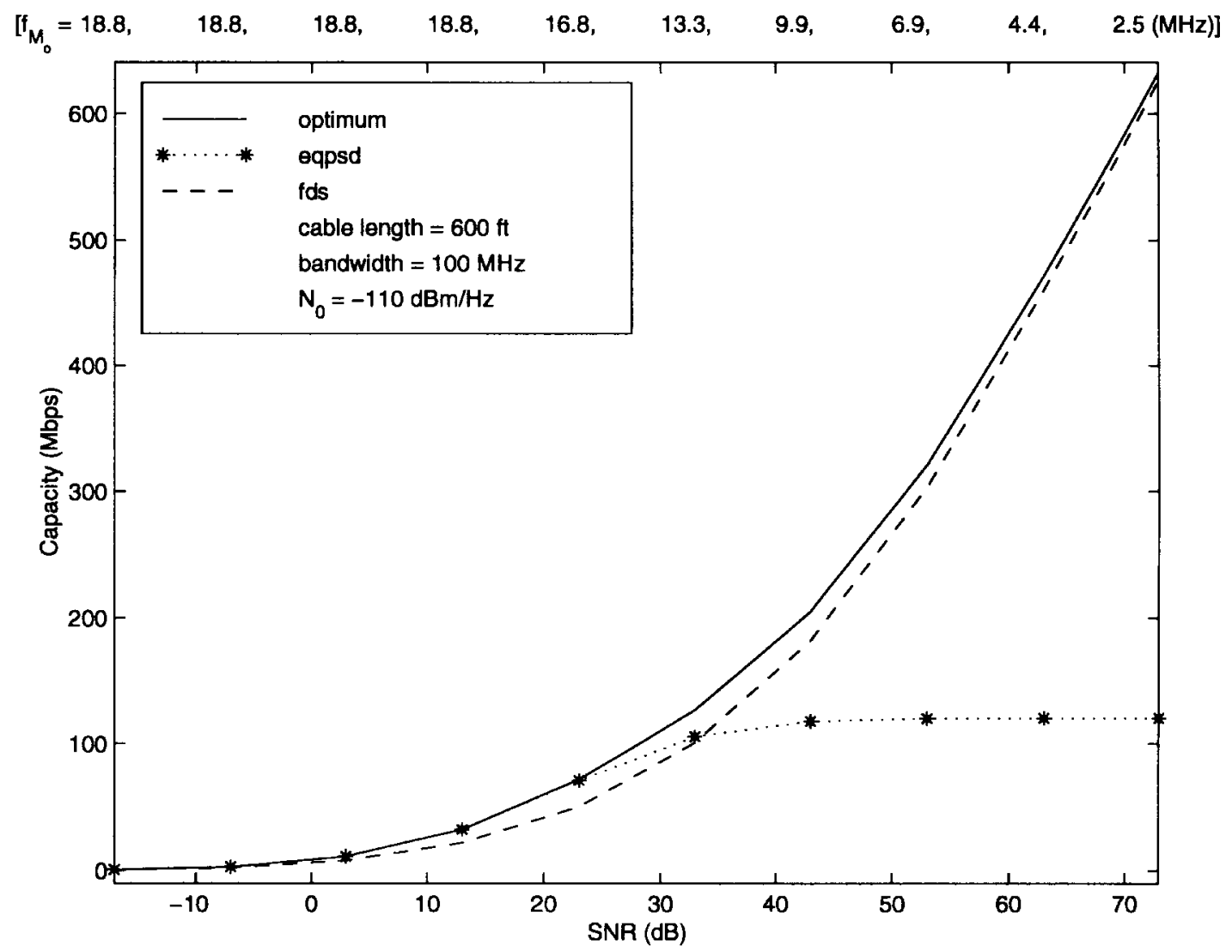

Fig. 6. Capacity calculations for twisted-pair model from [12]. The solid line corresponds to using the optimum transmission scheme (as described in Section IV), the star-dot line corresponds to using equal PSD's for the two directions of transmission (as assumed in [12]), and the dashed line corresponds to using FDS in all bins. The threshold frequency $f_{M_{0}}$ corresponding to each value of SNR is shown at the top of this figure. (The value of $f_{i *}$, where $\left(H_{i}-2 X_{i}\right) / X_{i}^{2}$ becomes negative, is $18.8 \mathrm{MHz}$.)

For the model of the twisted-pair channel used in [3]

$$
\left|H_{c}(f)\right|^{2}=\theta \exp (-\alpha \sqrt{f})
$$

where $\alpha$ is defined as in the previous model and $\theta$ represents the attenuation at $f=0$. Also

$$
\left|H_{x}(f)\right|^{2}=K f^{3 / 2}
$$

where $K=10^{-13}$. In the above expressions $f$ refers to frequency in hertz.

It is important to note that a closed-form expression for $\left|H_{c}(f)\right|^{2}$ was not given in [3]. However, the parameters $\alpha$ and $\theta$, in (16), were selected so that the resulting $\left|H_{c}(f)\right|^{2}$ matched the plot given in [3] as closely as possible. The fact that the model of $\left|H_{c}(f)\right|^{2}$ used in this example is not exactly equal to the one used in [3] is not important, since it is the approach to achieving capacity that is being tested, and this can accomplished using any monotonic model for $\left|H_{c}(f)\right|^{2}$. The capacity of this channel was calculated using $W=400$ $\mathrm{kHz}$ and $M=128$. The value used for $W$ corresponds to the one depicted in [3] and the value used for $M$ was chosen such that no significant change in calculated capacity resulted from increasing it. The resulting capacity, along with the corresponding threshold frequency $f_{M_{o}}$, are shown in Fig. 7 as functions of the SNR.

The curves plotted in Figs. 6 and 7 show the capacity under three different transmission schemes: the first uses equal
PSD's for all bins (just as in [12] and [3]), the second uses frequency division in all bins, and the third is the optimal, that is, it uses equal PSD's for the first $M_{o}$ bins (for some $\left.0 \leq M_{o} \leq M\right)$ and frequency division for the remaining bins. These correspond to the plots of $C_{\text {eqpsd }}\left(\{1, \cdots, M\}, P_{\max }\right)$, $C_{\mathrm{fds}}\left(\{1, \cdots, M\}, P_{\max }\right)$, and $C\left(P_{\max }\right)$, respectively, versus SNR. The value used for $\mathcal{N}_{O}$ in both plots is $-110 \mathrm{dBm} / \mathrm{Hz}$, which, as mentioned in [3], is a conservative value for AWGN power and is meant to account for a possible mismatch between the AWGN model and the actual distribution of the additive noise in the channel.

There are several observations that one can make about both figures. First, $C\left(P_{\max }\right)$ is larger than the other two for all values of SNR, as expected. More importantly, $C\left(P_{\max }\right)$ always increases with SNR, unlike $C_{\text {eqpsd }}$, which has a maximum value no matter how large the SNR becomes, as was also observed in [12] and [3].

Also, for low values of SNR, $C\left(P_{\max }\right)$ approaches $C_{\text {eqpsd }}$ and $f_{M_{o}}$ approaches $f_{i^{*}}$, implying that it is optimum to use equal PSD's in all bins that have a positive $\left(H_{i}-2 X_{i}\right) / X_{i}^{2}$. In addition, for high values of SNR, $C\left(P_{\max }\right)$ approaches $C_{\mathrm{fds}}$ and $f_{M_{o}}$ approaches zero, implying that it is optimum to use FDS in all bins. This result should be expected because at low SNR, crosstalk is not an important source of interference and thus both directions of transmission can use all of the frequency spectrum that has a positive value for $\left(H_{c}(f)-\right.$ 


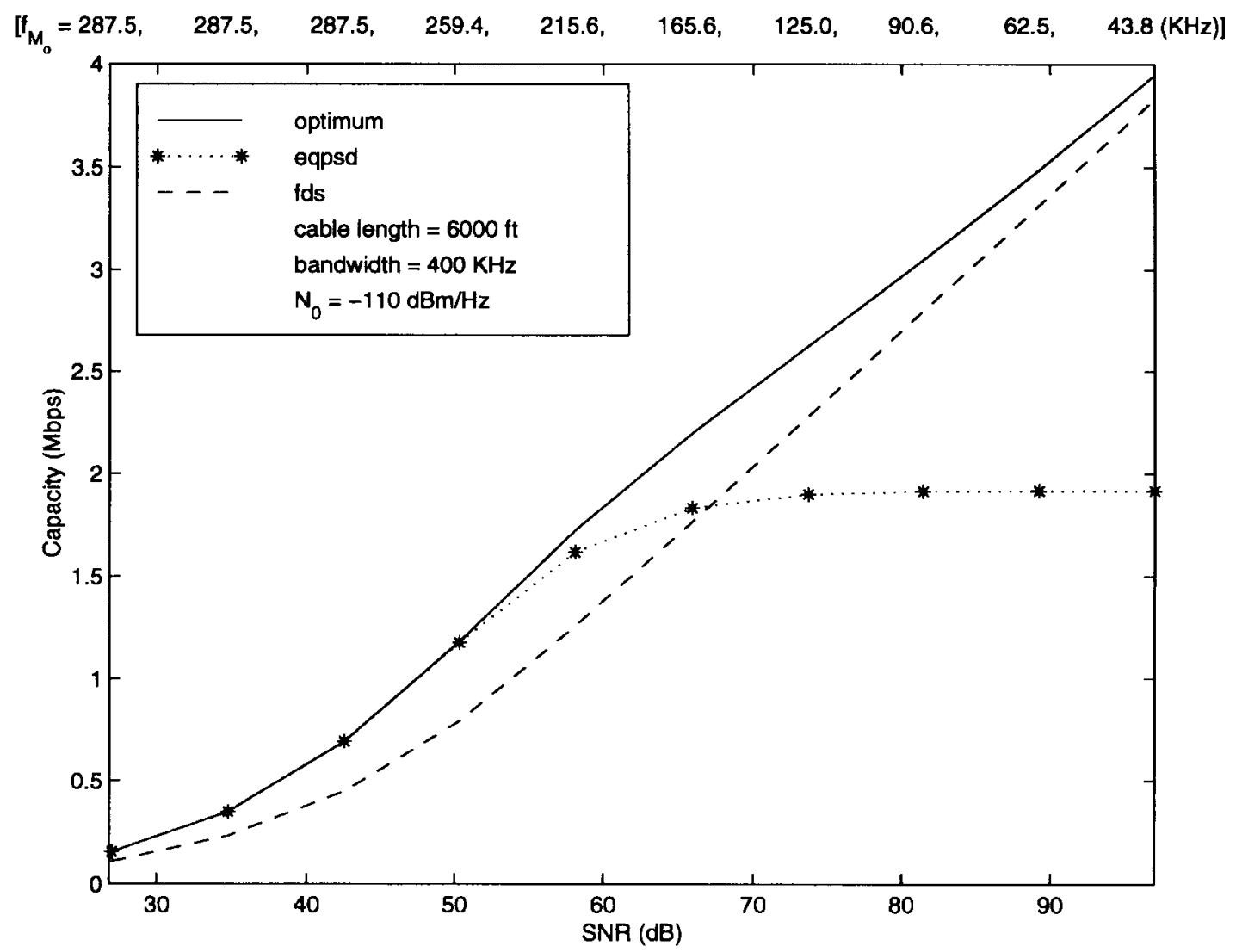

Fig. 7. Capacity calculations for twisted-pair model from [3]. The solid line corresponds to using the optimum transmission scheme (as described in Section IV), the star-dot line corresponds to using equal PSD's for the two directions of transmission (as assumed in [3]), and the dashed line corresponds to using FDS in all bins. The threshold frequency $f_{M_{O}}$ corresponding to each value of SNR is shown at the top of this figure. (The value of $f_{i *}$, where $\left(H_{i}-2 X_{i}\right) / X_{i}^{2}$ becomes negative, is $287.5 \mathrm{kHz}$.)

$\left.2 H_{x}(f)\right) / H_{x}^{2}(f)$. On the other hand, at very high SNR, crosstalk becomes a problem at all frequencies, implying that it should be eliminated using frequency division.

Finally, if $C\left(P_{\max }\right)$ is compared to $\max \left\{C_{\text {eqpsd }}, C_{\mathrm{fds}}\right\}$, it can be seen that it is not significantly larger at any SNR. In fact, the largest improvement that $C\left(P_{\max }\right)$ offers over $\max \left\{C_{\text {eqpsd }}, C_{\mathrm{fds}}\right\}$ is roughly $20 \%-30 \%$, and this occurs at moderate values of SNR. Therefore, if a $30 \%$ difference is not very important to the designer, the transmission system can be designed by calculating only $C_{\text {eqpsd }}$ and $C_{\mathrm{fds}}$ and using the scheme that offers higher capacity, thus saving computational time and effort.

It is important to discuss the results of [14], because it is the only work that questions the assumption that $S_{1}(f)$ has to be equal to $S_{2}(f)$ when deriving capacity of a twisted-pair channel. In [14] it is assumed that $\mathcal{N}_{0}=0$, that is, the only source of noise is NEXT. It is also assumed that $S_{1}(f)$ and $S_{2}(f)$ have the same support. Therefore, the resulting capacity is

$$
C_{1}=W \log _{2}\left(1+\left(\frac{H_{c}(f)}{H_{x}(f)}\right)_{f=f_{o}}\right)
$$

for some $f_{o} \in(0, W)$. It is clear that $C_{1}$, as defined in (17), is a finite quantity because $H_{x}(f)>0 \forall f \in(0, W)$. According to [14], this formula for capacity holds with no power constraints on the transmitted signals.
However, from (10) it follows that if the frequency range $0 \leq f \leq W$ is divided into $M$ bins, then FDS has to be used in all bins. Thus, each direction of transmission faces a channel with no noise. Since [14] assumes no power constraints on the transmitted signals, the resulting capacity is infinite.

It appears that this result is in contradiction with [14], where it was shown that the capacity is not only finite but only slightly better than the capacity when $S_{1}(f)$ is assumed to be equal to $S_{2}(f)$. The reason for this discrepancy may be that while [14] does not constrain $S_{1}(f)$ and $S_{2}(f)$ to be equal, it does constrain them to have the same support, a constraint we have not imposed.

\section{A. Bridge Taps}

As mentioned earlier, if the twisted-pair channel has bridge taps and other nonidealities, the channel and crosstalk transfer functions will, in general, not be monotonically decreasing and increasing, respectively. Therefore, Theorem 1 does not apply, implying that we need to solve the optimization problem described in either (11) or (13). For small values of $M$, both approaches are computationally viable. However, as $M$ increases, they both become computationally prohibitive, especially the approach in (13). Interestingly, though, just as in the monotonic channel case, it is the approach in (13) that can be reduced to a significantly simpler problem. This will be shown via numerical examples. 

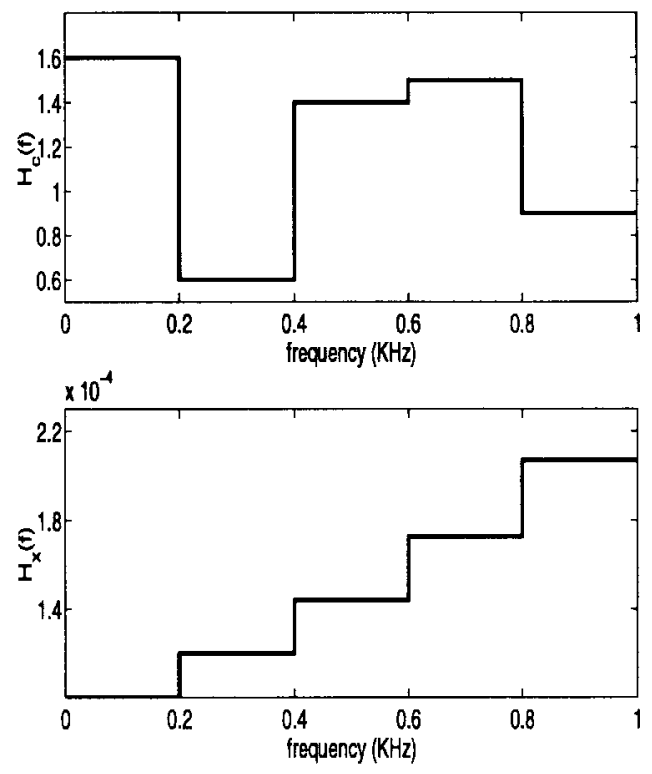

(a)

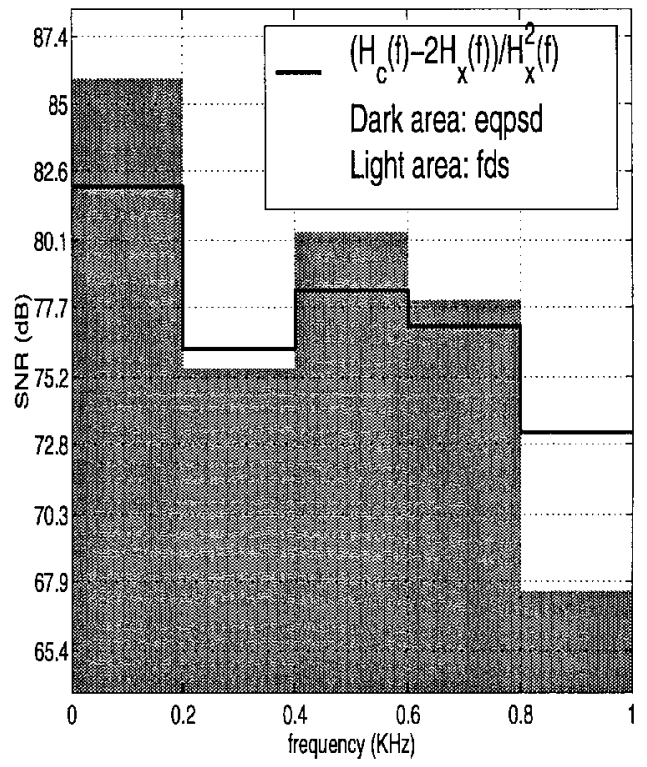

(b)

Fig. 8. (a) Channel and crosstalk transfer functions of the five-bin channel. (b) Signaling strategies as a function of the SNR. At each value of SNR, equal PSD's are used in all of the frequency ranges (bins) that are shaded, and frequency division is used in the remaining frequency ranges (bins). Also, the value of $\left(H_{c}(f)-2 H_{x}(f)\right) / H_{x}^{2}(f)$ as a function of frequency is depicted as a solid line.

For the purposes of understanding the properties of optimal signaling strategies for nonmonotonic channels, we first look at a simple channel that consists of five piecewise constant segments. The channel and crosstalk transfer functions are depicted in Fig. 8(a). The capacity of this channel was calculated using both (11) and (13). What is of interest is not the capacity of this fictitious channel but the resulting pattern of signaling schemes used in each bin, as a function of the SNR. This pattern is shown in Fig. 8(b).

A second channel that was studied was the one in [3], however with a null artificially added to the channel transfer function at $60 \mathrm{kHz}$. For $M=128$, the optimization problem described in (11) is still solvable, though extremely timeconsuming. Again, what is of interest is not the capacity of this channel but the resulting pattern of signaling schemes used in each bin, as a function of the SNR. This pattern is shown in Fig. 9.

We see in Figs. 8 and 9 that the optimal signaling strategies exhibit the following behavior. At very low SNR's, all bins with a positive $\left(H_{i}-2 X_{i}\right) / X_{i}^{2}$ employ equal PSD's and all bins where this quantity is negative employ frequency division. As the SNR increases, we see that the equal PSD regions become interspersed by pockets of frequency-division bins. These pockets form in the parts of the spectrum where the nulls in $\left(H_{i}-2 X_{i}\right) / X_{i}^{2}$ are. As the SNR increases even further, these pockets of frequency-division bins become wider and wider until all bins employ frequency division. This behavior was also observed in numerous other examples that were tested but are not included here. Such behavior suggests a methodology for solving the general optimization problem posed in (13). For example, if $\left(H_{c}(f)-2 H_{x}(f)\right) / H_{x}^{2}(f)$ has only one null, then (15) can be easily extended as given in (18), shown at the bottom of the page.

That is, compared to the monotonic channel case, we have added two more points where the signaling strategy changes, to account for the null. Although, the computational complexity of this problem is slightly larger than that of (15), it is significantly smaller than that of (13). Unfortunately, this approach suffers from the drawback that for each additional null found in $\left(H_{c}(f)-2 H_{x}(f)\right) / H_{x}^{2}(f)$, two additional threshold points need to be added to the optimization in (18). This not only increases the complexity of the optimization but also introduces implementation difficulties because of the ambiguity in the number of nulls that exist in a given channel.

These problems are alleviated if we interpret the behavior of the optimal signaling strategies in a different way. The behavior at very low SNR's is as described above. However, as the SNR increases, an increasing number of bins switch their signaling strategies over to frequency division. Moreover, this switching seems to occur in an orderly fashion as seen in Figs. 8 and 9 . That is, bins with lower values for $\left(H_{i}-2 X_{i}\right) / X_{i}^{2}$ switch over to frequency division before bins with a higher

$$
C\left(P_{\max }\right)=\max _{\substack{M_{1}, M_{2}, M_{3}, P_{1} \\ \text { s.t. } 0 \leq M_{1} \leq M_{2} \leq M_{3} \leq M \\ 0 \leq P_{1} \leq P_{\max }}} C_{\text {eqpsd }}\left(\left\{i \mid i \in\left(0, M_{1}\right] \cup\left(M_{2}, M_{3}\right]\right\}, P_{1}\right)+C_{\mathrm{fds}}\left(\left\{i \mid i \in\left(M_{1}, M_{2}\right] \cup\left(M_{3}, M\right]\right\}, P_{\max }-P_{1}\right)
$$




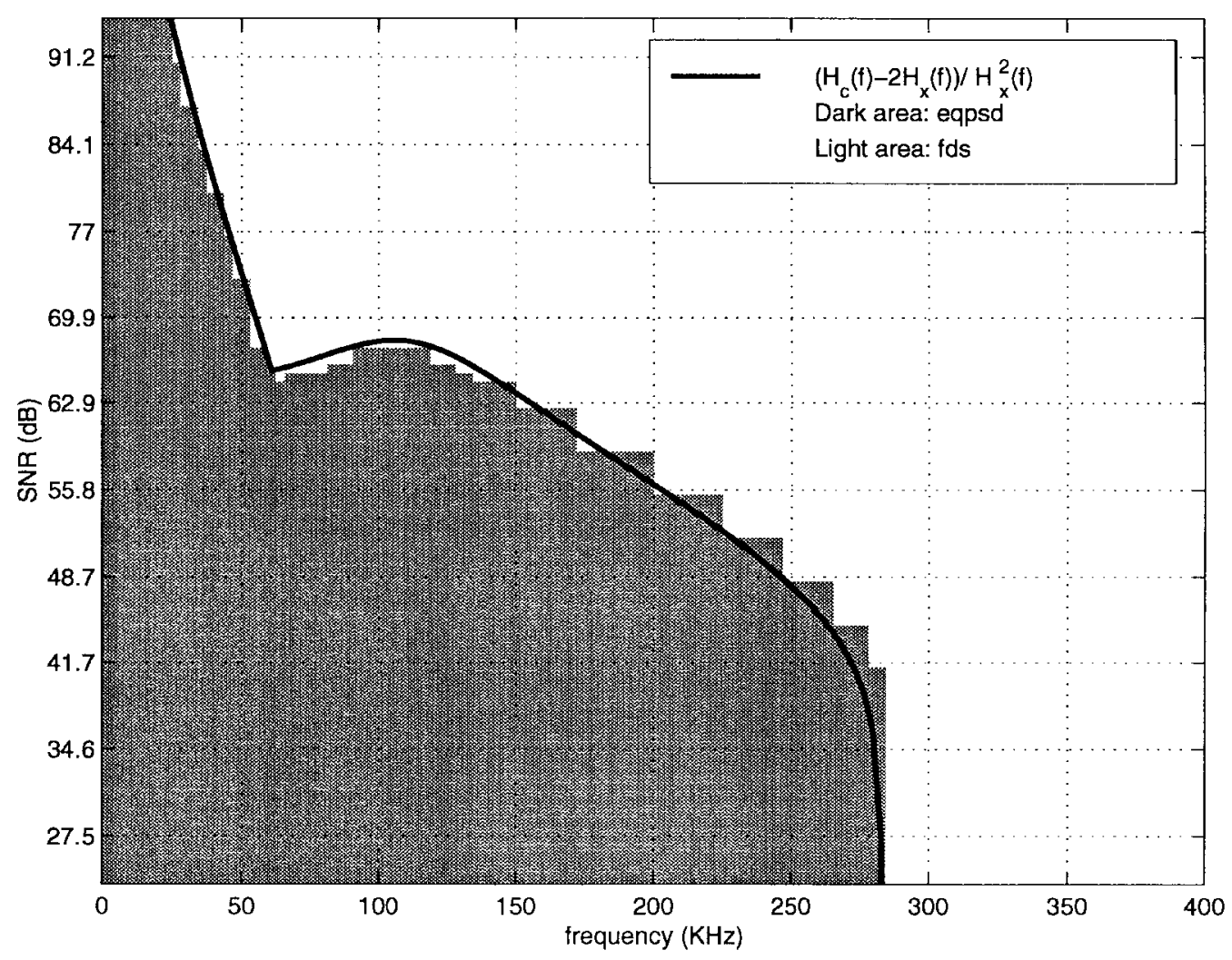

Fig. 9. Signaling strategies as a function of the SNR for the channel in [3] with a null added at $60 \mathrm{kHz}$. At each value of SNR, equal PSD's are used in all of the frequency ranges (bins) that are shaded, and frequency division is used in the remaining frequency ranges (bins). Also, the value of $\left(H_{c}(f)-2 H_{x}(f)\right) /\left(H_{x}^{2}(f)\right)$ as a function of frequency is depicted as a solid line. (The value of $f_{i^{*}}$, where $\left(H_{i}-2 X_{i}\right) / X_{i}^{2}$ becomes negative, is $287.5 \mathrm{kHz}$.)

value for this quantity. Eventually, at high enough SNR's all bins employ frequency division. This behavior suggests an alternative methodology for solving the general optimization problem posed in (13). That is, we first sort $\left(H_{i}-2 X_{i}\right) / X_{i}^{2}$ in descending order and then relabel the channel and crosstalk transfer functions as $\tilde{H}_{i}$ and $\tilde{X}_{i}$ such that $\left(\tilde{H}_{i}-2 \tilde{X}_{i}\right) / \tilde{X}_{i}^{2}$ is a decreasing function of $i$. Thus, (15) is extended as given in (19), shown at the bottom of the page, where $\tilde{C}_{\text {eqpsd }}$ and $\tilde{C}_{\text {fds }}$ are defined as before, except for the fact that they refer to the relabeled channel described by $\tilde{H}_{i}$ and $\tilde{X}_{i}$. This approach has the advantages of having a low computational complexity [in fact, the same as that of (15)] and of not requiring the ambiguous task of determining the number of nulls in the channel.

Of course, we have not established that either of the above approaches is optimal since we have not yet been able to prove that the behavior of the optimal signaling strategies described above is exhibited in all twisted-pair channels. However, they do yield solutions which, for the examples considered, agree with the solutions to (11), given in Figs. 8 and 9.

As as a final comment, we note that the results shown in Fig. 9 lead us to make an even stronger statement about the behavior of the optimal signaling strategies. Since the shaded area in the figure follows the plot of $\left(H_{c}(f)-\right.$ $\left.2 H_{x}(f)\right) / H_{x}^{2}(f)$ fairly closely, it appears that we may be able to approximate the optimal signaling strategy, and thus obtain a lower bound on the capacity, by requiring all bins for which $2 P_{\max } / \mathcal{N}_{o} W \leq\left(H_{i}-2 X_{i}\right) / X_{i}^{2}$ to employ equal PSD's and all of the remaining bins to employ frequency division. Notice that the above condition involves the total SNR, and not the SNR in the $i$ th bin, which, of course, would have been optimal. This strategy reduces to the following simple optimization problem:

$$
C\left(P_{\max }\right)=\max _{\substack{P_{\mathcal{A}} \\ \text { s.t. } 0 \leq P_{\mathcal{A}} \leq P_{\max }}} C_{\text {eqpsd }}\left(\mathcal{A}, P_{\mathcal{A}}\right)+C_{\mathrm{fds}}\left(\mathcal{A}^{\mathcal{C}}, P_{\max }-P_{\mathcal{A}}\right)
$$

where $\mathcal{A}$ is the set of indexes of all bins for which $2 P_{\max } / \mathcal{N}_{o} W \leq\left(H_{i}-2 X_{i}\right) / X_{i}^{2}$. It turns out that the resulting capacity is very close to the one obtained using the optimal signaling strategy, while at the same time requiring the

$$
C\left(P_{\max }\right)=\max _{\substack{M_{o}, P_{1} \\ \text { s.t. } 0 \leq M_{o} \leq M \\ 0 \leq P_{1} \leq P_{\max }}} \tilde{C}_{\text {eqpsd }}\left(\left\{i \mid i \in\left(0, M_{o}\right]\right\}, P_{1}\right)+\tilde{C}_{\mathrm{fds}}\left(\left\{i \mid i \in\left(M_{o}, M\right]\right\}, P_{\max }-P_{1}\right)
$$




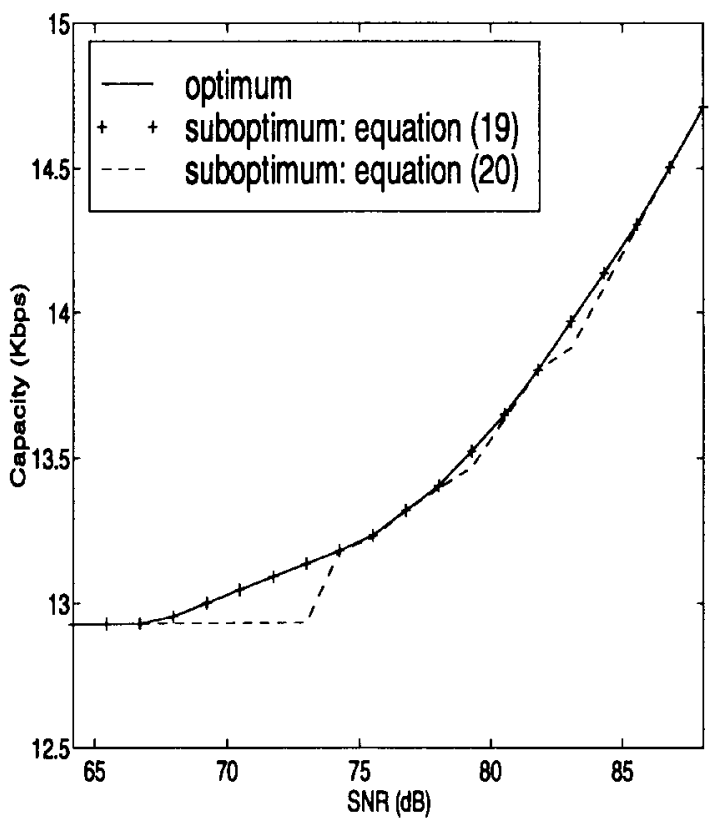

(a)

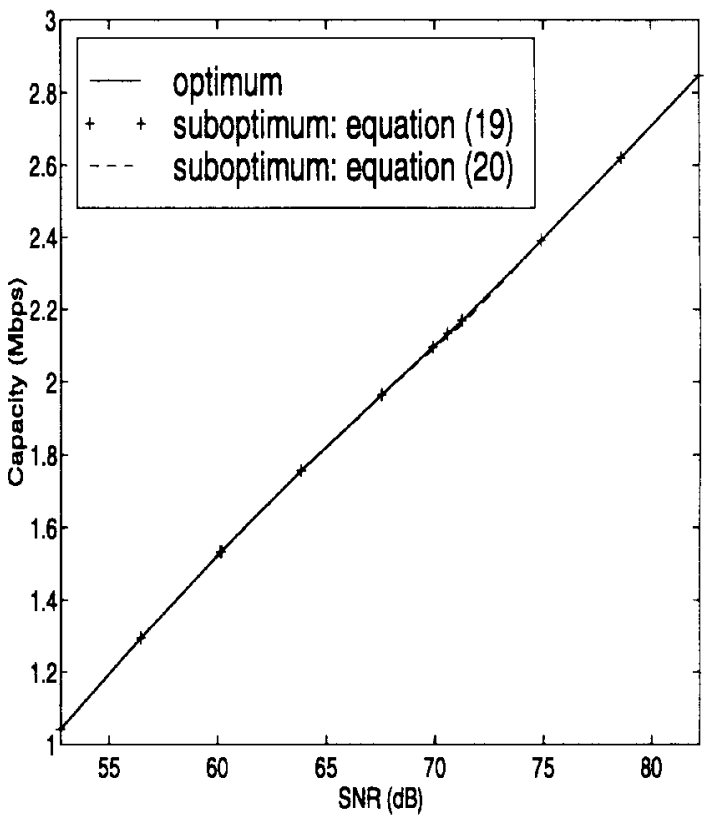

(b)

Fig. 10. Capacity as a function of the SNR for various signaling schemes. (a) For channel of Fig. 8. (b) For channel of Fig. 9.

solution of a problem with an extremely lower computational complexity than either (11) or (13).

The values of the capacity versus SNR resulting from the optimal signaling scheme, which is described in (11), and the two suboptimal schemes described by (19) and (20), are plotted in Fig. 10 for the two channels used in Figs. 8 and 9. It can be seen that the results corroborate our analysis and discussion. Notice how the capacity resulting from (19) is identical to that resulting from (11), which is optimal. Also notice that the capacity resulting from (20), although less than the optimal, still follows the optimal capacity fairly closely, especially in Fig. 10(b).

\section{CONCLUSION}

We established that allowing different PSD's for the two directions of transmission over twisted-pair channels and performing a joint optimization over these PSD's can result in significant increases in the information capacity. In particular, we showed that the capacity is unbounded as the SNR goes to infinity. For monotonic channels, the optimal signaling strategies that we derived have a simple threshold structure--the signal PSD's in the two directions are identical up to a threshold frequency, beyond which they occupy disjoint frequency slots. Our numerical results also indicate how the optimal strategies get modified for nonmonotonic channels.

The optimal signal PSD's derived in this paper may be achieved in practice using a number of methods. However, the structure of the signal PSD's lends itself to a straightforward implementation using multicarrier modulation [17]-[23]. For example, when the channel has monotonic channel and crosstalk transfer functions, the first $M_{o}$ carriers should be assigned to both directions of transmission while the remaining carriers should be assigned alternatingly to each direction. Also, the appropriate power (and bit rate) should be used for each carrier so that the signal PSD is as desired.

An interesting topic for further research is the extension of Theorem 1 to channels with nonmonotonic channel response and crosstalk transfer functions. The results presented in Section V should provide a starting point for this research. Also of interest is the analysis of the actual bit rate achievable by a multicarrier signaling scheme that uses the optimal PSD's derived in this paper. Another avenue for further research is the capacity analysis under peak power constraints. Finally, since the analysis here does not apply directly to ADSL, a possibility for future work would be the application of the approach used in this paper to ADSL systems.

\section{REFERENCES}

[1] J. W. Lechleider, "High bit rate digital subscriber lines: A review of HDSL progress," IEEE J. Select. Areas Commun., vol. 9, pp. 769-784, Aug. 1991.

[2] J.-J. Werner, "The HDSL environment," IEEE J. Select. Areas Commun., vol. 9, pp. 785-800, Aug. 1991.

[3] J. T. Aslanis and J. M. Cioffi, "Achievable information rates on digital subscriber loops: Limiting information rates with crosstalk noise," IEEE Trans. Commun., vol. 40, pp. 361-372, Feb. 1992.

[4] D. L. Waring, "The asymmetrical digital subscriber line (ADSL): A new transport technology for delivering wideband capabilities to the residence," in IEEE GLOBECOM'91, vol. 3, Phoenix, AZ, pp. 1979-1986, Dec. 1991.

[5] Integrated Services Digital Network (ISDN)—Basic Access Interface for Use on Metallic Loops for Application on the Network Side of the NT (Layer 1 Specification), American National Standard for Telecommunications, American National Standards Institute, Feb. 1992.

[6] D. G. Messerschmitt, "Echo cancelation in speech and data transmission," IEEE J. Select. Areas Commun., vol. SAC-2, pp. 283-297, May 1984.

[7] W. Chen, J. L. Dixon, and D. L. Waring, "High bit rate digital subscriber line echo cancelation," IEEE J. Select. Areas Commun., vol. 9, pp. 848-860, Aug. 1991.

[8] J. Yang, S. Roy, and N. H. Lewis, "Data driven echo cancelation for a multitone modulation system," IEEE Trans. Commun., vol. 42, pp. 2133-2144, May 1994. 
[9] J. Cioffi and J. A. C. Bingham, "A data-driven multitone echo canceler," IEEE Trans. Commun., pp. 2853-2869, Oct. 1994.

[10] M. Ho, J. M. Cioffi, and J. A. C. Bingham, "Discrete multitone echo cancelation," IEEE Trans. Commun., vol. 44, p. 817-825, 1996.

[11] T. M. Cover and J. A. Thomas, Elements of Information Theory. New York: Wiley, 1991.

[12] I. Kalet and S. Shamai, "On the capacity of a twisted-wire pair: Gaussian model," IEEE Trans. Commun., vol. 38, pp. 379-383, Mar. 1990.

[13] G.-H. Im and J. J. Werner, "Bandwidth-efficient digital transmission up to $155 \mathrm{mb} / \mathrm{s}$ over unshielded twisted pair wiring," in IEEE Int. Conf. Communications '93, Geneva, Switzerland, May 1993, pp. 17971803.

[14] J. W. Lechleider, "The capacity of NEXT-impaired subscriber loops," in IEEE GLOBECOM'90, vol. 2, San Diego, CA, pp. 1161-1165, Dec. 1990.

[15] K. J. Kerpez, "Near-end crosstalk is almost Gaussian," IEEE Trans. Commun., vol. 41, pp. 670-672, May 1993.

[16] A. Sendonaris, V. Veeravalli, and B. Aazhang, "Joint signaling strategies for maximizing the capacity of twisted pair loops," Rice Univ., Houston, TX, Tech. Rep. 9508, July 1995, Available HTTP: http://wwwece.rice.edu/ $\sim$ sendos.

[17] I. Kalet, "The multitone channel," IEEE Trans. Commun., vol. 37, pp. 119-124, Feb. 1989

[18] J. A. C. Bingham, "Multicarrier modulation for data transmission: An idea whose time has come," IEEE Commun. Mag., vol. 28, pp. 5-14, May 1990.

[19] A. K. Khandani, P. Kabal, and H. Leib, "Combined coding and shaping over a multitone channel," in IEEE GLOBECOM'91, vol. 2, Phoenix, AZ, Dec. 1991, pp. 1182-1186.

[20] J. S. Chow, J. C. Tu, and J. M. Cioffi, "A discrete multitone transceiver for HDSL applications," IEEE J. Select. Areas Commun., vol. 9, pp. 895-908, Aug. 1991.

[21] P. S. Chow, J. C. Tu, and J. M. Cioffi, "Performance evaluation of a multichannel transceiver system for ADSL and VHDSL services," IEEE J. Select. Areas Commun., vol. 9, pp. 909-919, Aug. 1991.

[22] T. N. Zogakis, J. T. Aslanis, and J. M. Cioff, "Coded and shaped discrete multitone system," IEEE Trans. Commun., vol. 43, pp. 2941-2949, Dec. 1995.

[23] S. D. Sandberg and M. A. Tzannes, "Overlapped discrete multitone modulation for high speed copper wire communications," IEEE J. Select. Areas Commun., vol. 13, pp. 1571-1585, Dec. 1995.

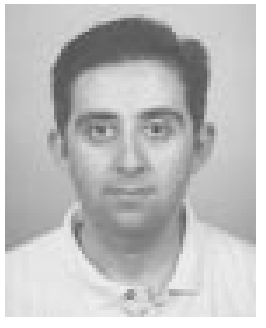

Andrew Sendonaris (S'93) received the B.S. (summa cum laude) and M.S. degrees in electrical engineering from Rice University, Houston, TX, in 1993 and 1995, respectively. He is currently working toward the Ph.D. degree at Rice University.

His current research interests include multipleaccess communications over fading multipath channels, code-division multiple-access (CDMA) systems, the capacity of, and resource allocation for, cellular mobile radio networks, and digital transmission over twisted-pair copper loops.

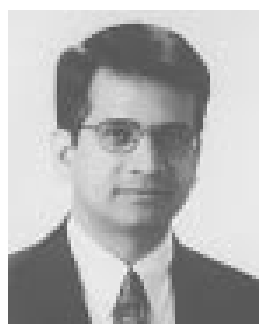

Venugopal V. Veeravalli (S'84-M'93) received the B. Tech. degree from the Indian Institute of Technology, Bombay, (Silver Medal Hons.), in 1985, the M.S. degree from Carnegie-Mellon University, Pittsburgh, PA, in 1987, and the Ph.D. degree from the University of Illinois, Urbana-Champaign, in 1992, all in electrical engineering.

He joined Cornell University, Ithaca, NY, in 1996, where he is currently an Assistant Professor of electrical engineering and a Member of the Graduate Fields of statistics and applied mathematics. He has been with Harvard University, Cambridge, MA, as a Postdoctoral Fellow, the City College of New York, NY, as an Assistant Professor, and Rice University, Houston, TX, as a Visiting Assistant Professor. He teaches and conducts research in telecommunication systems, mobile and wireless communications, detection and estimation theory, and information theory. Current topics of research include design and analysis of code-division multiple-access (CDMA) systems, capacity analysis for wireless communication systems, channel modeling and error control coding for wireless systems, resource allocation in wireless networks, decentralized dynamic decision making, sequential multihypothesis testing, and sensor fusion. He has also served as Consultant on several industry projects, particularly in the wireless communications area.

Dr. Veeravalli is a corecipient of the 1996 IEEE Browder J. Thompson Award and a recipient of a 1998 National Science Foundation CAREER award.

Behnaam Aazhang (S'82-M'85-SM'91), for photograph and biography, see p. 399 of the March 1998 issue of this Transactions. 